\title{
Eyecciones coronales de masa solar a 1 unidad astronómica
}

Maribel S. Guerrero Vásquez ${ }^{1}$

\section{RESUMEN}

La presente investigación concluye estadísticamente que las eyecciones coronales de masa solar (CME) por sus siglas en inglés, son grandes erupciones de campos magnéticos coronales solares y plasma (considerado el cuarto estado de la materia), que alcanzan la órbita de la Tierra con características especiales de velocidad, en este caso específico estudiado, se convierten en tormentas geomagnéticas, de alta 0 baja velocidad, según las $\mathrm{CME}$.

Para desarrollar la presente investigación se estudiaron los datos de eyecciones coronales de masa solar con propiedades delimitadas: de velocidad, masa y diferentes tipos de energía del año 1990 al 2012 que pudieron convertirse en tormentas geomagnéticas. Se enfocó la investigación en eventos rápidos con un rango de tiempo mayor o igual a 0.5 días y menor o igual a 1 día, para calcular el tiempo de llegada al planeta Tierra en días; se usó la distancia promedio entre nuestro planeta y el Sol.

De los datos de CME de altas velocidades, tenemos como resultados pocas fluctuaciones del campo magnético, o sea, pocas tormentas geomagnéticas; pero se observaron valores de índices $\mathrm{k}$ desde 0 a 9 . En general, haciendo un análisis comparativo de los resultados de la caracterización de eyecciones coronales con la actividad solar, se observó que los ciclos de máxima actividad fueron atípicos, concluyendo que realmente los ciclos mínimos se han prolongado, se resalta la tendencia decreciente en la actividad solar y como consecuencia también hay tendencia decreciente en la ocurrencia de eyecciones coronales de masa que son la principal causa de las tormentas geomagnéticas.

Palabras claves: tormentas geomagnéticas, eyecciones coronales de masa, plasma, ondas de choque, velocidad, masa, energía, Sol, actividad, satélites.

\footnotetext{
${ }_{1}^{1}$ Profesora universitaria, Facultad de Ciencias Espaciales, UNAH: marsuy3000@yahoo.com
} 


\section{ABSTRACT}

This research concludes that statistically Coronal Mass Ejections sun (CME for its acronym in English), are large eruptions of solar coronal magnetic fields and plasma (considered the fourth state of matter) that reach the Earth's orbit, with special features to velocity in this specific case study, become Geomagnetic Storms, whether high or low velocity. To develop these research data coronal mass ejections of solar, with defined properties were studied; speed, mass, and different types of energy from 1990 to 2012, which could become geomagnetic storms. Research is focused on speed events with a range of greater than or equal to 0.5 days and less than or equal to one days time to calculate the time of arrival to Earth in days, using the average distance between Earth and the sun. From data CME speeds; results have as few fluctuations of the magnetic field, ie few geomagnetic storms; but $k$ indexes values were observed from 0 to 9 . In general making a comparative analysis of the results of the characterization of CMEs with solar activity, it was observed that the peak cycles were atypical, concluding that actually the minimum cycles they have long, downward trend in solar activity is highlighted and as a result there are decreasing trend in the occurrence of coronal mass ejections are the main cause of geomagnetic storms.

Keywords: geomagnetic storms and coronal mass ejections (CME), shock waves, speed, mass, power, sun, satellites 


\section{INTRODUCCIÓN}

Figura 1. Varios aspectos del Sol

A

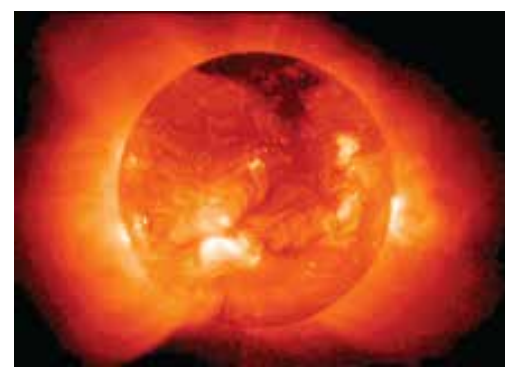

B

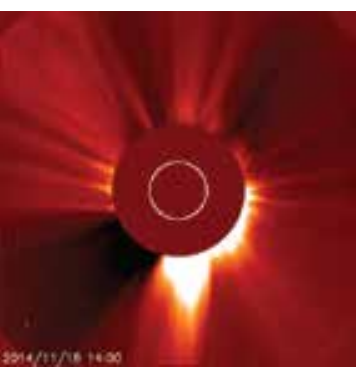

C

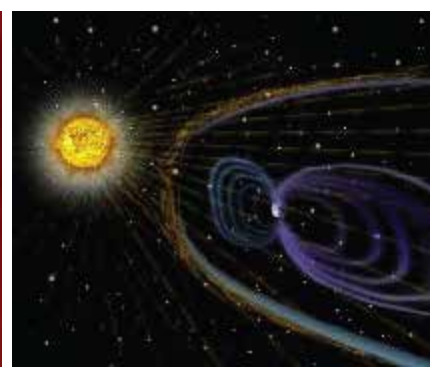

Aimagen de la corona solar en rayos X, emitiendo radiación. B. LASCO C2 (cronógrafo 2) muestra una eyección de masa coronal (CME). C. La imagen artística de la protección de la magnetosfera y la influencia del Sol (cortesía NASA- ESA).

La importancia de poder predecir el clima global de la Tierra de acuerdo al clima espacial, es un objetivo implícito en toda investigación de la física solar y su relación con este. Del comportamiento del clima de la Tierra se desprende la relevancia social y económica de mitigar posibles consecuencias globales por daños en la tecnología satelital, entre otros.

Con las observaciones de varios satélites en la actualidad se registran continuamente durante 24 horas, algunos de los fenómenos en la corona solar (ver figura 1A), como las CME (ver figura 1B) en diferentes longitudes de onda; a la vez ocasionan variabilidad en el clima espacial y tormentas geomagnéticas, que son perturbaciones temporales de la magnetosfera terrestre (ver figura $1 \mathrm{C}$ ). La radiación que penetra la atmósfera forma las auroras boreales y australes que son los indicadores visibles de la presencia real de la radiación recibida.

El efecto de la llegada al planeta Tierra de más o menos radiación del Sol, es evidente que influencia directamente la variabilidad climática, reflejada en la temperatura como indicador del dominio del Sol. Las CME son ondas de choque que llegan entre 24 y 36 horas después del suceso, esto solamente ocurre si la onda de choque viaja hacia la Tierra. La presión sobre la magnetosfera aumentará o disminuirá en función de la actividad solar, ya que la presión modifica las corrientes eléctricas en la ionosfera. 
Las tormentas magnéticas duran de 24 a 48 horas, aunque pueden prolongarse varios días; así, los cambios en los campos geomagnéticos afectan negativamente la atmósfera superior y la baja atmósfera, destruyen células vivientes y producen cambios climáticos y las partículas muy energéticas afectan las partes electrónicas de los satélites; asimismo, las grandes tormentas geomagnéticas causan cambios en su órbita, provocando la caída o pérdida de estos.

\section{METODOLOGÍA}

De las mediciones de los fenómenos naturales, en este caso fenómenos solares como las eyecciones coronales solares de masa (CME), con sus variables escogidas aquí como la velocidad, aceleración, masa y diferentes tipos de energía escogidas; las tormentas geomagnéticas con sus indicadores los índices K, o sea, las mediciones del campo magnético terrestre tocado entre otras necesarias para concretar la investigación cualitativamente y cumplir con el objetivo de correlacionar las CME con tormentas geomagnéticas.

Se buscaron, seleccionaron y archivaron los datos digitales satelitales del satélite Solar and Heliospheric Observatory ( $\mathrm{SOHO}$ ), las $\mathrm{CME}$ y del medio interplanetario que llegan a la Tierra para transformarse en tormentas geomagnéticas. Tal la información corresponde a los años 1996 al 2012 y describe las características de los datos observados antes mencionados. Luego, se hicieron los cálculos de velocidades promedio, entre las velocidades lineales y velocidades de segundo orden.

Para calcular el tiempo de llegada al planeta en días de cada evento, se utilizó la distancia promedio entre la Tierra y el Sol $(149,500,000 \mathrm{~km})$. Se construyeron tablas de CME con altas velocidades para enfocar la investigación en eventos rápidos con un rango de tiempo mayor o igual a 0.5 días y menor o igual a 1 día. Consecuentemente, se elaboraron las tablas de tormentas geomagnéticas moderadas, fuertes, severas y extremas, correlacionadas en el tiempo de llegada a la Tierra y las eyecciones coronales solares que se produjeron para recorrer 1 unidad astronómica. Se computaron las estadísticas de los datos de la época seleccionada, se graficaron esos resultados y por último se concluyó con el análisis de los resultados estadísticos. 


\section{RESULTADOS}

Tabla 1. CME Y TG 1996- 2012

\begin{tabular}{|c|c|c|c|c|c|}
\hline Años & $\begin{array}{c}\text { Cantidad de CME } \\
\text { encontradas }\end{array}$ & $\begin{array}{c}10 \text { selección } \\
\text { CME }\end{array}$ & $\begin{array}{c}\mathbf{0 . 5 3} \text { dias }<\text { T } \\
\text { Llegada CME } \\
\text { caracterizadas } \\
<=<=1 \text { día }\end{array}$ & $\%$ halos & $\begin{array}{c}\text { Tormentas } \\
\text { geomagnéticas } \\
\text { encontradas }\end{array}$ \\
\hline $\begin{array}{c}1996- \\
2004\end{array}$ & 9943 & 458 & 61 & 77 & 13,140 \\
\hline $2005-$ & 9190 & 156 & 19 & 88 & 11,680 \\
\hline 2012 & & 80 & $83 \%$ & 24,820 \\
\hline Total & & & & & \\
\hline
\end{tabular}

En la tabla 1, se observa la cantidad de eyecciones coronales solares (CME) acontecidas que se reportaron desde el año 1996 a 2012, en la página web del satélite $\mathrm{SOHO}, 9943 \mathrm{CME}$, de las que se seleccionaron 458 con velocidad mayor a $450 \mathrm{~km} / \mathrm{s}$, que es la velocidad promedio del viento solar que llega en aproximadamente en 4 días a la órbita de la Tierra.

Dentro del rango de tiempo planificado discriminante en esta investigación, mayor o igual 0.53 días y menor o igual a 1 día, que salieron al espacio interplanetario en un total de 80 CME, 61 entre 1996 - 2004 y 19 CME del 2005 a 2012. Aunque se esperaría que los halos (cuando las CME sobrepasan los 1800 en el campo de visión del telescopio) fuesen proporcionales a la cantidad de eyecciones coronales, resultaron sorprendentes las cifras, debido a que no hay eventos solo de halos con la regla discriminatoria de velocidad, explicada arriba. Los resultados muestran que hubo más CME rápidas entre 1996 y 2004 (61), que de 2005 a 2012 (19).

Asimismo, la cantidad de tormentas geomagnéticas (columna 6 TABLA 1) arroja valores mayores que el de las $\mathrm{CME}$, lo cual significa que otras $\mathrm{CME}$ menos rápidas quedaron fuera de las estadísticas por no cumplir el requerimiento de tener una velocidad mayor a $450 \mathrm{~km} / \mathrm{s}$, que llegaron en forma más lenta al planeta, pero que finalmente tocaron su campo magnético. 
Tabla 2. Coincidencias CME y TG moderadas, fuertes, severas y extremas $1996-2012$

\begin{tabular}{|c|c|c|c|c|c|}
\hline \multicolumn{2}{|c|}{$\begin{array}{l}\text { Cantidad de CME } \\
\text { encontradas }\end{array}$} & \multicolumn{4}{|c|}{$\begin{array}{l}\text { Resumen coincidencias CME (20 selección )- } \\
\text { tormentas geomagnéticas (índice } \mathbf{k} \text { ) }\end{array}$} \\
\hline Años & & $\begin{array}{c}\mathrm{K}=6 \mathrm{G} 2 \\
\text { Tormentas } \\
\text { moderadas }\end{array}$ & $\begin{array}{c}\mathrm{K}=7 \mathrm{G} 3 \\
\text { Tormentas } \\
\text { fuertes }\end{array}$ & $\begin{array}{c}\mathrm{K}=8 \mathrm{G} 4 \\
\text { Tormentas } \\
\text { severas }\end{array}$ & $\begin{array}{c}\text { K=9 G5 } \\
\text { Tormentas } \\
\text { Extremas }\end{array}$ \\
\hline $\begin{array}{l}1996- \\
2004\end{array}$ & 9943 & 24 & 9 & 9 & 3 \\
\hline $\begin{array}{r}2005- \\
2012\end{array}$ & 9190 & 13 & 10 & 4 & 3 \\
\hline Total & & 37 & 19 & 13 & 6 \\
\hline
\end{tabular}

En la tabla 2, se muestran las tormentas geomagnéticas más peligrosas expresadas por los índices $\mathrm{K}$ con los valores más altos establecidos de 6 a 9 , cabe recordar que los índices utilizados son los valores reportados para altas latitudes. Se observa que los acontecimientos van en orden decreciente se presentaron en total 13 severas, 19 extremas y 37 moderadas; solo 6 tormentas muy peligrosas extremas con índice k=9 y correspondiente a $500 \mathrm{nT}$ (nano Teslas). Acontecieron estas tormentas peligrosas en las CME lentas y muy rápidas, dentro de la baja actividad solar de los últimos años.

Tabla 3. Caracterización de CME a tormentas geomagnéticas

\begin{tabular}{lllllll} 
Caracterización & $\begin{array}{l}\text { Ángulo de } \\
\text { posición } \\
\text { centra }\end{array}$ & $\begin{array}{l}\text { Amplitud } \\
\text { angular } \\
\text { (grados) }\end{array}$ & $\begin{array}{l}\text { Veloc. } \\
\text { lineal } \\
\mathbf{k m} / \mathbf{s}\end{array}$ & $\begin{array}{l}\text { Veloc. de } \\
\text { segundo } \\
\text { orden } \\
\text { final }[\mathbf{k m} / \mathbf{s}]\end{array}$ & $\begin{array}{l}\text { Veloc. } \\
\text { promedio } \\
{[\mathbf{k m} / \mathbf{s}]}\end{array}$ & $\begin{array}{l}\text { Tiempo } \\
\text { de Ilegada } \\
\text { a la Tierra } \\
\text { en días }\end{array}$ \\
$\begin{array}{l}\text { Valores } \\
\text { máximos }\end{array}$ & Halo (OA) & 360 & 3387 & 3284 & 3264.5 & 0.5300 \\
\hline $\begin{array}{l}\text { Valores } \\
\text { mínimos }\end{array}$ & 2 & 24 & 218 & 280 & 278 & 1.005 \\
\end{tabular}




\begin{tabular}{llllll}
\hline Caracterización & $\begin{array}{l}\text { Tiempo de } \\
\text { llegada a } \\
\text { la Tierra } \\
\text { en horas }\end{array}$ & $\begin{array}{l}\text { Veloc. a } \\
\mathbf{2 0} \text { radios } \\
\text { solares }[\mathbf{k m} / \mathbf{s}]\end{array}$ & $\begin{array}{l}\text { Aceleración } \\
\mathbf{k m} / \mathbf{s} 2\end{array}$ & $\begin{array}{l}\text { Masa } \\
\text { [gramos] }\end{array}$ & $\begin{array}{l}\text { Energía } \\
\text { cinética (ergios) }\end{array}$ \\
$\begin{array}{llllll}\text { Valores } \\
\text { máximos }\end{array}$ & 16.30 & 1124 & $198.0^{* 1}$ & $1.9 \mathrm{e}+32$ & 359 \\
\hline $\begin{array}{l}\text { Valores } \\
\text { mínimos }\end{array}$ & 24.12 & -179.7 & -159.1 & $2.70 \mathrm{e}+29$ & 15 \\
\end{tabular}

En la tabla 3, se aprecian las características principales para que las eyecciones coronales solares puedan llegar a tocar el campo magnético de la Tierra, o sea, convertirse en tormentas geomagnéticas (TG). En forma resumida, se observa que el valor máximo para ángulos de posición central es un halo $\mathrm{OA}$ (con esquema de asimetría), por lo tanto, su amplitud angular es igual a 360 grados.

Cada CME, se caracteriza por tres velocidades: la velocidad lineal (o ajuste polinomial) obtenida ajustando una línea recta, para las mediciones en tiempo; la velocidad cuadrática obtenida mediante el ajuste de una parábola (cuadrática o de segundo orden ajuste polinomial) para las mediciones en tiempo altura y la evaluación de la velocidad en el momento final (última posible medida de la altura) y la velocidad obtenida como en el valor mínimo, del ángulo de posición central 2, (Tabla 3) pero evaluada cuando la CME está a una distancia de 20 radios solares.

Desde el momento de la medición de la altura final, esta varía de un evento a otro, la velocidad de 20 radios solares es útil para comparar diferentes velocidades. Se debe tener precaución en el tratamiento de las CME que se desvanecen antes de llegar a 20 radios solares. Su velocidad lineal $3387 \mathrm{~km} / \mathrm{s}$ es la más alta encontrada en el rango de tiempo estudiado de 1996-2012 y la velocidad de segundo orden es muy alta igual a $3284 \mathrm{~km} / \mathrm{s}$. El promedio de ambas resultó igual a $3264.5 \mathrm{~km} / \mathrm{s}$.

Conociendo la velocidad promedio y la distancia promedio se calculó el tiempo promedio que tardó en llegar esa eyección de masa coronal a la Tierra, cuyo resultado es de 0.53 días, o sea, 16.3 horas. Otra característica es la velocidad de la CME a 20 radios solares de distancia más allá de la órbita de Urano, antes de Neptuno, aún sigue siendo un evento rápido. 
La aceleración de una CME puede ser positiva, negativa o cercana a cero, lo que significa que una CME se acelera, se mueva con velocidad constante o disminuye la velocidad en la observación del telescopio de LASCO. Así, se necesita un mínimo de tres mediciones en tiempo de altura para una estimación de la aceleración, pero la precisión aumenta cuando hay más mediciones. Las aceleraciones con solo tres mediciones no son confiables, por lo tanto, no se reportan datos. En la tabla 3, columna 10, se aprecia una aceleración alta, positiva igual a $1980 \mathrm{~km} / \mathrm{s} 2$.

Cada CME, también se caracteriza por una masa y una energía cinética. En general, existen grandes incertidumbres en estos números. La estimación de la masa de una CME implica una serie de supuestos, por eso los valores dados deben ser tomados como representativos. Por ejemplo, la mayoría de las CME muestran un aumento de la masa cuando atraviesan los primeros radios solares y a continuación la masa alcanza un valor casi constante. Este valor constante se toma como la masa representativa, para este caso vemos en la columna 11 (Tabla 3), el dato de la masa para el evento más rápido igual a $1.9 \mathrm{e}+32$ gramos y la energía cinética igual a 359 ergios. El 10 de noviembre de 2004 se reportaron tormentas con índices $\mathrm{k}$ de 6,7 y 8 , ninguna con $\mathrm{k}$ igual a 9 .

Para el evento más lento (velocidad promedio $278 \mathrm{~km} / \mathrm{s}$ ) dentro del rango de tiempo definido como máximo (1 día) para llegar a nuestro planeta, se encontraron otras características que muestra la tabla 3 , columna dos, en donde se ve que el ángulo de posición central es de 2 grados y la amplitud angular es apenas de 24 grados, la masa es grande y la energía cinética muy baja. Pero, que finalmente llegó a la Tierra y se pudo convertir en tormenta geomagnética.

En la figura 1, se muestra la CME más rápida ocurrida el 10 de noviembre de 2004, del coronógrafo $\mathrm{C} 2$ de LASCO a bordo del satélite $\mathrm{SOHO}$, sus respectivas gráficas de velocidad, ángulo de posición y la aceleración negativa, es decir, que hubo desaceleración (-159.1 km/s2) posiblemente debido a su gran masa, entre otras variables. Penosamente en los meses de abril, mayo, junio, julio, agosto y septiembre no hubo ningún tipo de registro del NOAA. 
Figura 2. Imagen de una CME a las 02:48 UT, con la velocidad máxima encontrada en los datos desde 1996 al 2012 del coronógrafo C2 en infrarrojo extremo

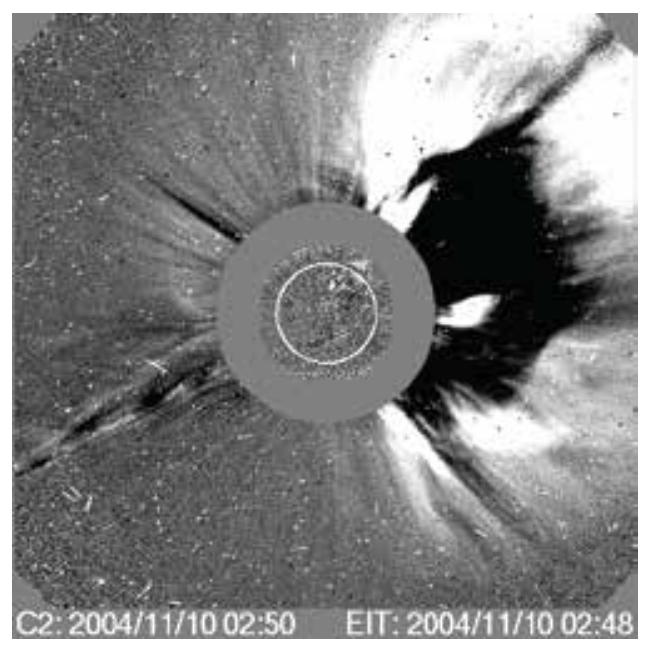

Gráficos de la CME con velocidad máxima VMAX =3,387km $/ \mathrm{s}, 2004$ / 11 / 10, ángulo de posición y aceleración del evento

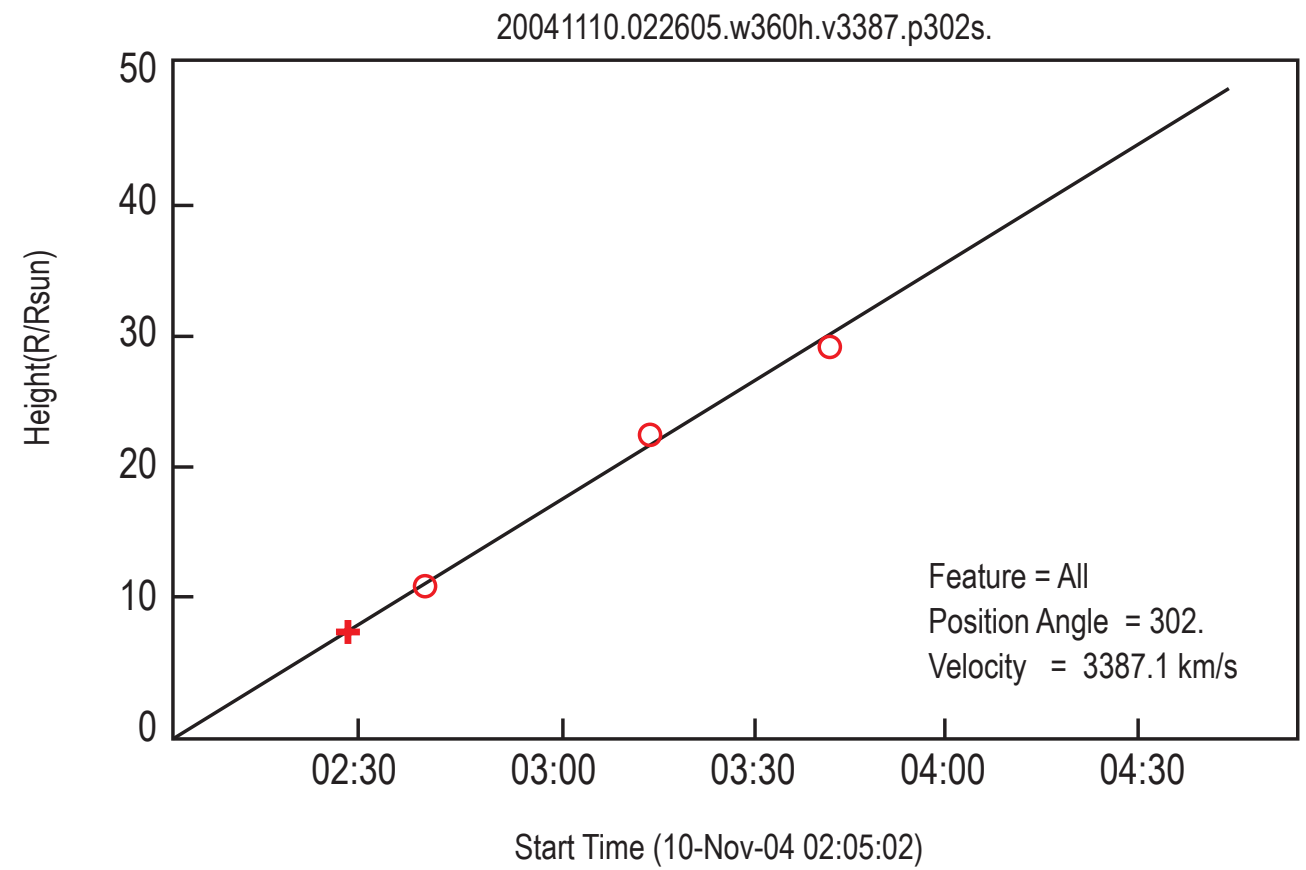




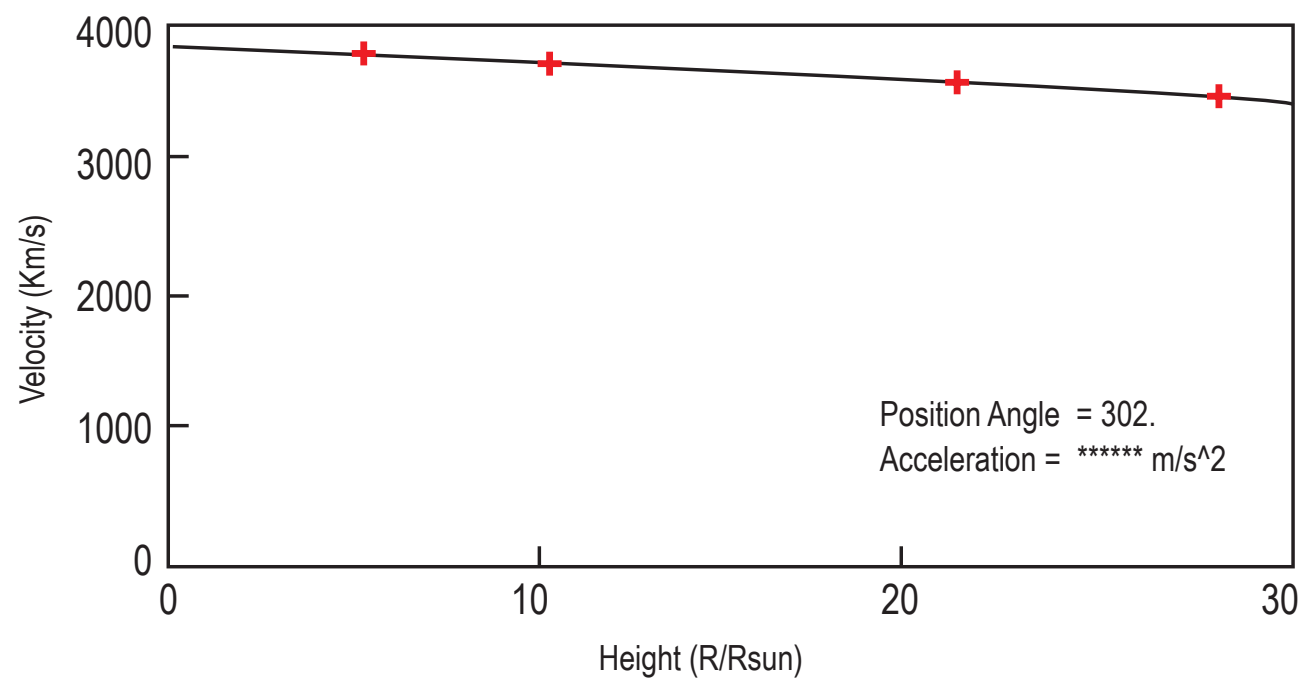

Figura 3. La gráfica superior. muestra la velocidad lineal y ángulo de posición, en la imagen central se ve la gráfica del tiempo de inicio, la inferior muestra el ángulo de posición y la aceleración del mismo evento.

Imagen del C2 del 2012/07/0611:48 UT

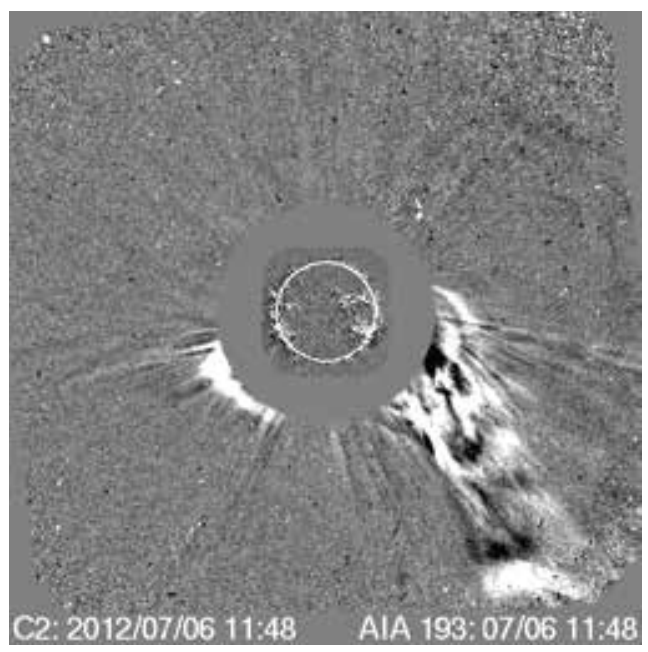


Figura 4. Muestra la CME a las 11:48 UT, de mínima velocidad encontrada

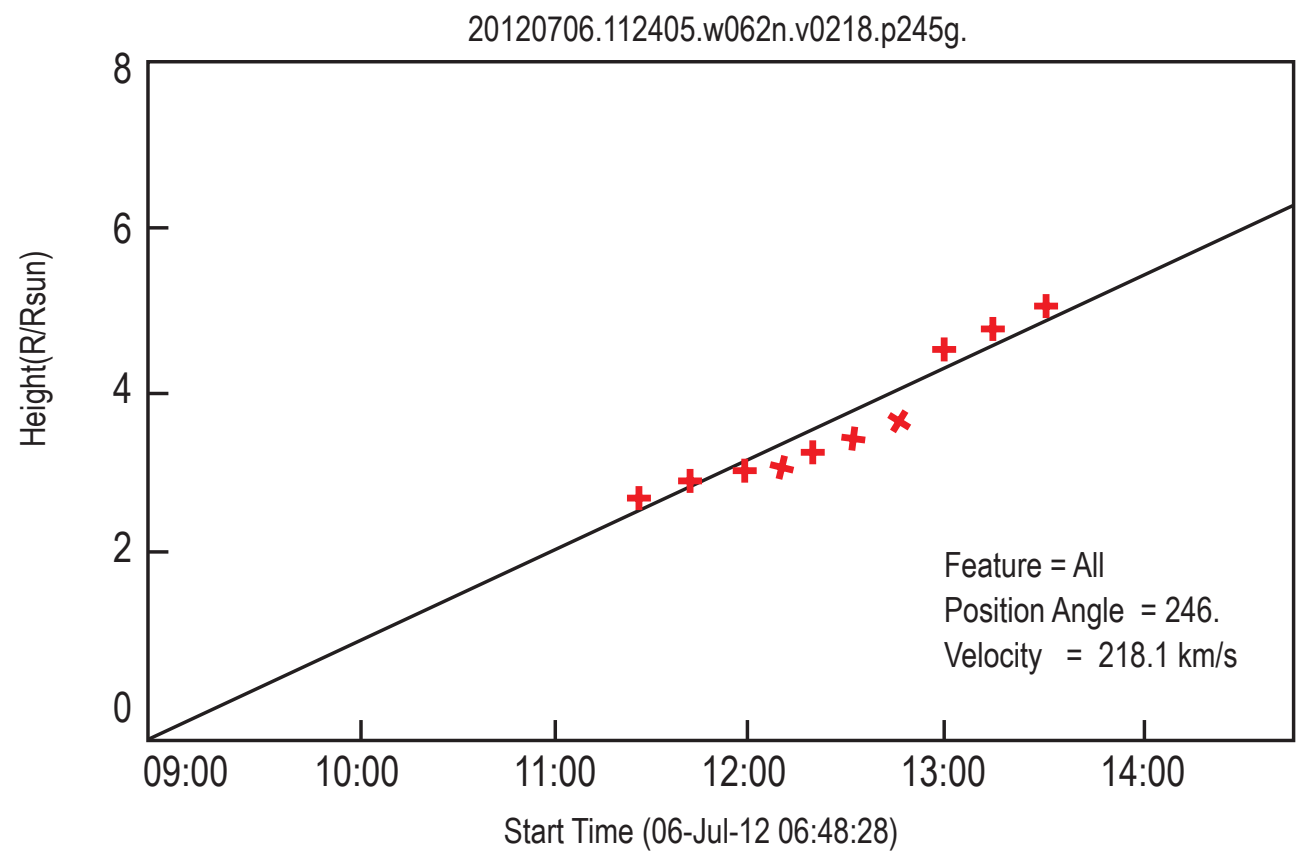

Figura 5. CME del 2012/07/06, de mínima velocidad encontrada, Vlineal MIN=218km/

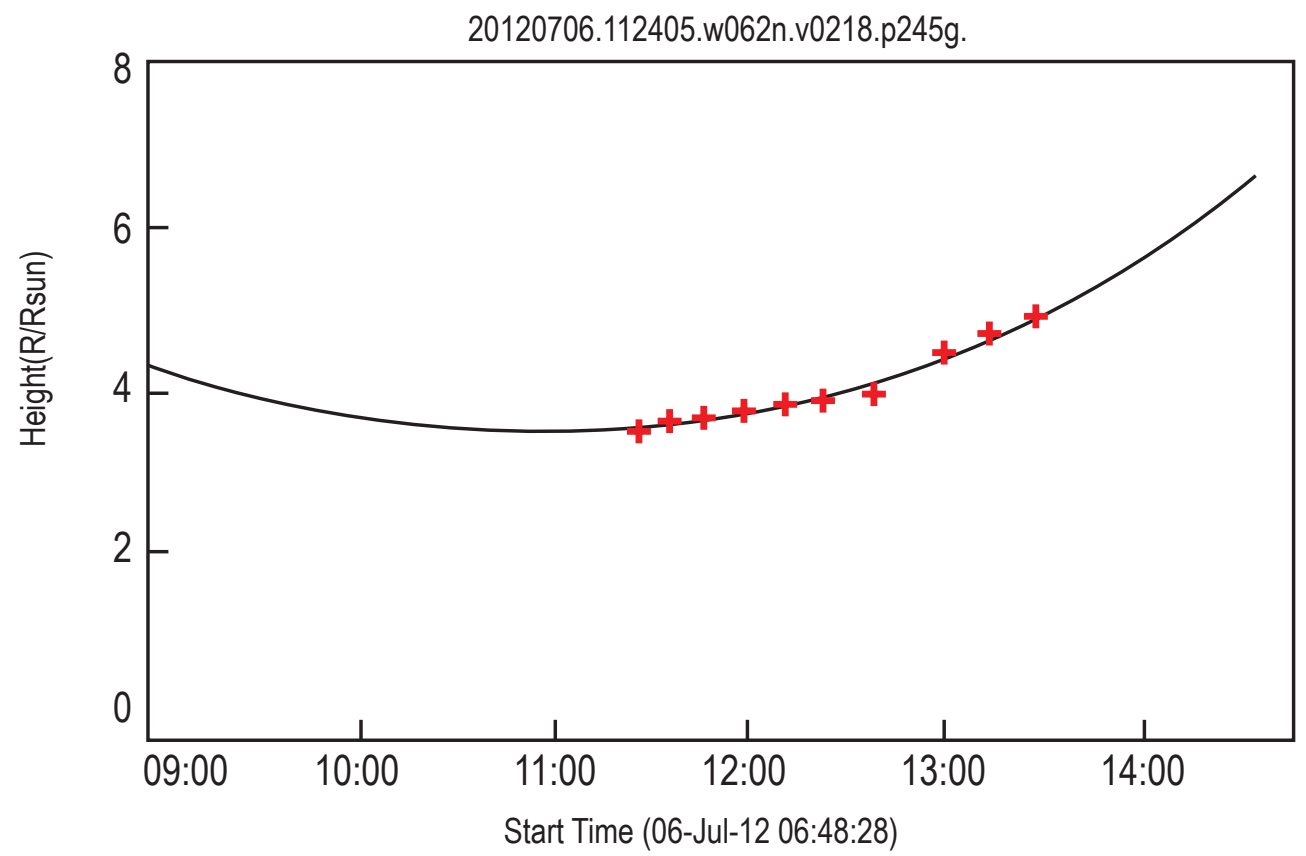




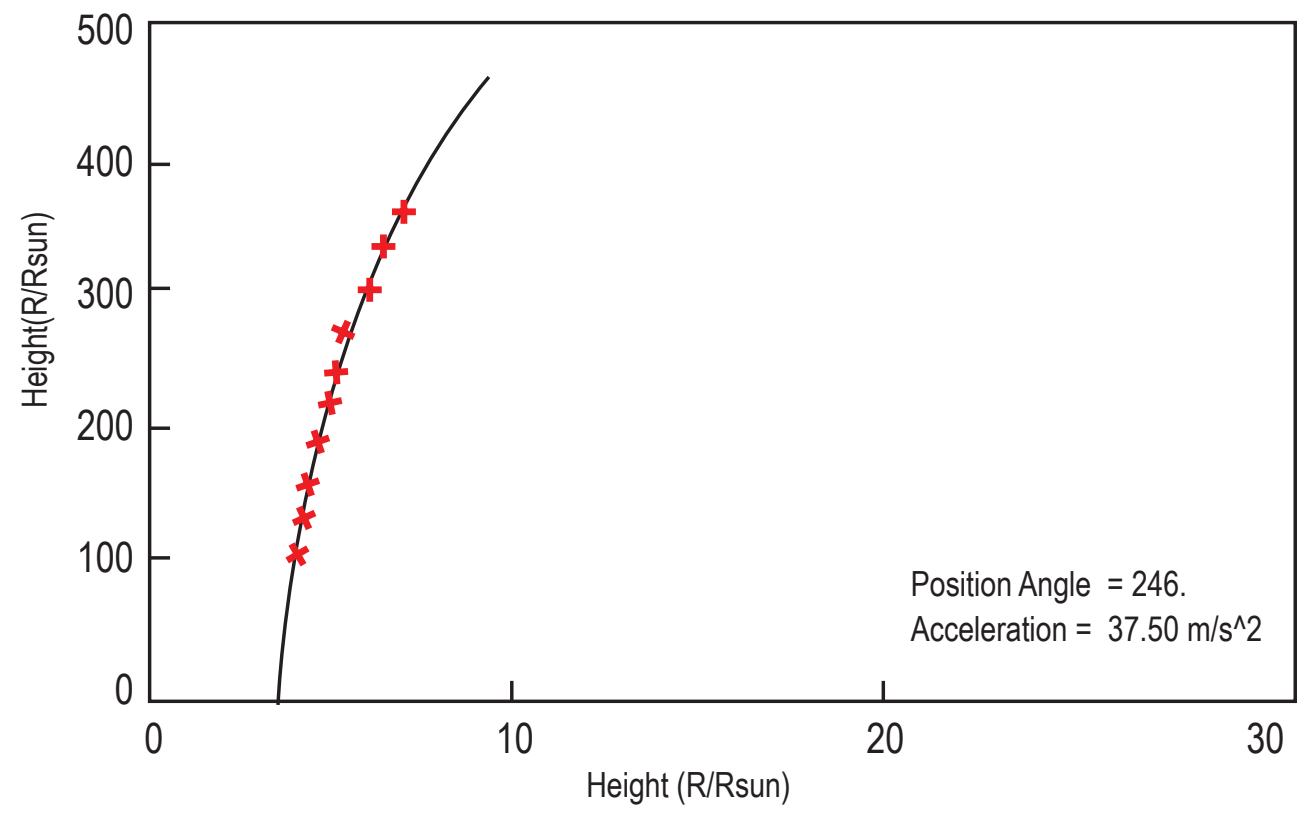

El grafico, muestra la Vlinea IMIN=218 km/s el grafico del centro se ve la curva del tiempo de inicio de la CME; la inferior representa la velocidad de segundo orden a 20 radios solares.

\section{DISCUSIÓN}

Un análisis comparativo muestra los resultados de la caracterización de eyecciones coronales de masa solar con la actividad solar, en donde los ciclos de máxima actividad fueron atípicos, se presentó mayor actividad en los ciclos mínimos y viceversa, concluyendo que los ciclos mínimos (ver figuras 6 y 7 ) se han prolongado y realmente no ha habido máxima actividad, por eso se ha llamado máximos a los dos últimos por definición: por la posición cercana de las manchas respecto al ecuador del Sol, como consecuencia, tenemos pocas fluctuaciones del campo magnético, o sea, pocas tormentas geomagnéticas y escasos eventos solares en general, durante los periodos llamados de máxima actividad solar.

Pero, se observaron valores de índices $\mathrm{k}$ desde 0 a 9 . En forma específica para la 
CME máxima, vista por primera vez a las 2:26 horas, se sumó el tiempo aproximado de 0.53 días que tardó en llegar a la Tierra, entonces, se correlaciona con la tormenta geomagnética con índice $k=7$, ocurrida entre las $12-15$ horas reportadas. De la CME más lenta estudiada no se puede concluir nada, porque no hay datos del satélite NOAA en esa fecha. El estudio de la caracterización también se vio afectado por la escasez de eventos muy rápidos y también lentos, en la época de investigación definida. Con los elementos estudiados se puede decir que toda eyección de masa coronal rápida que llega a la Tierra produce disturbios del campo magnético, del orden de índice k igual o mayor a 7.

Figura 6. La imagen muestra la baja actividad del Sol. Desde el 2011, existe un número de manchas solares muy por debajo de los valores previstos y las fuertes erupciones solares que se esperaban han sido muy poco frecuentes, asegura el observatorio de la NASA.

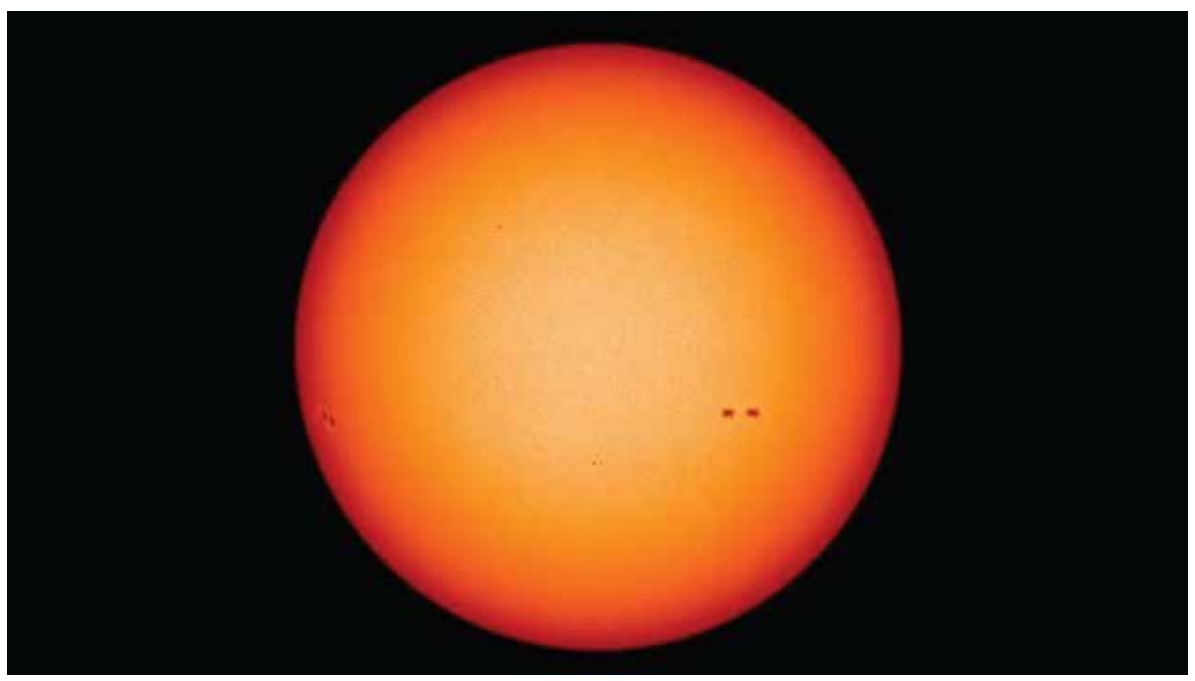

Baja actividad del Sol 
Figura 7. Arriba la curva muestra la tendencia de tormentas geomagnéticas desde 1989 al presente; en la imagen inferior la curva representa la tendencia lineal y polinomial descendente debido a la baja actividad del Sol (Guerrero, 2012).

Comportamiento y Tendencia de la actividad solar

\section{Comportamiento de las tarmentas geomagnéticas en los ciclos Máximos y Mínimos}

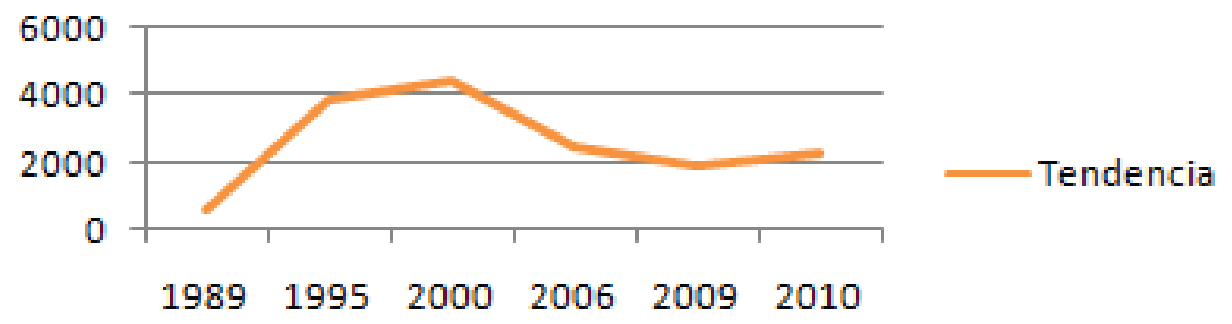

Cantidad de tormentas geomagnéticas (TG) desde 1989 al presente y la curva de tendencia polimonal

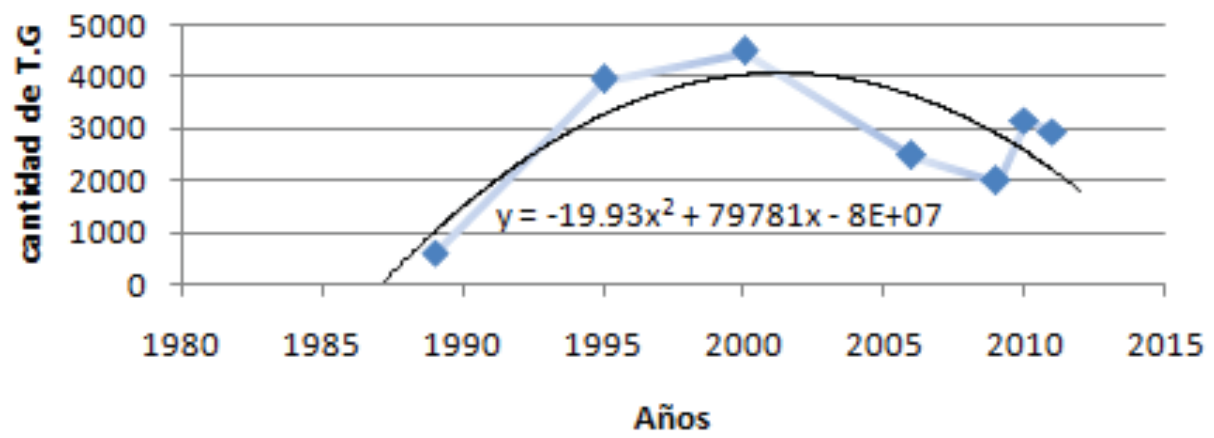


Tabla 4. Resumen de coincidencias CME -tormentas geomagnéticas (índice k)

\begin{tabular}{|c|c|c|c|c|c|}
\hline \multicolumn{2}{|c|}{$\begin{array}{l}\text { Cantidad de CME } \\
\text { encontradas }\end{array}$} & \multicolumn{4}{|c|}{$\begin{array}{l}\text { Resumen coincidencias CME -tormentas } \\
\text { geomagnéticas (índice } \mathbf{k} \text { ) }\end{array}$} \\
\hline Años & & $\begin{array}{c}\mathrm{K}=6 \mathrm{G} 2 \\
\text { Tormentas } \\
\text { moderadas }\end{array}$ & $\begin{array}{c}\mathrm{K}=7 \mathrm{G} 3 \\
\text { Tormentas } \\
\text { fuertes }\end{array}$ & $\begin{array}{c}\text { K=8 G4 } \\
\text { Tormentas } \\
\text { severas }\end{array}$ & $\begin{array}{c}\text { K=9 G5 } \\
\text { Tormentas } \\
\text { Extremas }\end{array}$ \\
\hline $\begin{array}{l}1996- \\
2004\end{array}$ & 9943 & 24 & 9 & 9 & 3 \\
\hline $\begin{array}{r}2005- \\
2012\end{array}$ & 9190 & 13 & 10 & 4 & 3 \\
\hline Total & & 37 & 19 & 13 & 6 \\
\hline
\end{tabular}

\section{CONCLUSIONES}

En conjunto, esta investigación tiene por objeto proporcionar un análisis cuantitativo completo y específico, con la descripción de todos los aspectos principales de un evento máximo único y un evento mínimo único que comenzaron con una CME y que se esperaba que tocara la magnetosfera, convirtiéndose en una tormenta geomagnética con un valor cualquiera de la medición del índice $\mathrm{K}$ en altas latitudes, pero que pudieran tener mayor posibilidad, los valores más altos para $\mathrm{k}$ iguales a 7,8 y 9 por sus características arriba descritas.

De lo anterior se puede inferir la generalización: que todas las eyecciones coronales de masa que fueron seleccionadas por ser muy rápidas, en principio, y con masas relativamente bajas terminaron convirtiéndose en tormentas geomagnéticas con valores de índices $\mathrm{k}$ desde 0 hasta 9 , desde tormentas menores hasta extremas. Concluyendo que no dependen de la velocidad para ser tormentas peligrosas, pero que el clima del Sol afecta directamente al clima de la Tierra y otros planetas, es quien lo controla, subiendo o bajando su temperatura de acuerdo a la actividad máxima 0 mínima de la estrella más cercana. 


\section{BIBLIOGRAFÍA}

Auzmendi, E. (1992). Las actitudes hacia la matemática-estadística en las enseñanzas medias y universitaria. España: Bilbao y Mensajero.

Baron, R. y Byrne, D. (2005). Psicología social. Madrid: Pearson Educación.

Carifio, J. y Perla, R. (2008). Revising the 50 year debate around using and misusing Likert scales. Medical Education, 42(12), 1150-1152.

Coetzee, S. y Van Der Merwe, P. (2010). Industrial Psychology Student's Attitudes Towards Statistics. SA Journal of Industrial Psychology, 36(1), 1-8.

Garcia, J.; Fallas, M. y Romero, A. (2015). Las actitudes hacia la estadística del estudiantado de orientación. Revista Electrónica Educare, 19(1), 25-41.

Hernández, R.; Fernández, C. y Baptista, P. (2010). Metodología de la investigación. Perú: McGraw-Hill.

Kaplan, R. y Saccuzzo, D. (2006). Pruebas psicológicas. Méjico: Thomson.

Murray, J. (2013). Likert data: What to use, Parametric or Non-Parametric. International Journal of Business and Social Science, 4(11), 258-264.

Ramírez, C.; Schau, C. y Emmioglu, E. (2012). The Importance of Attitude in Statistics Education. Statistics Education Research Journal, 11(2), 57-71.

Sandoval, C. (1996). Investigación cualitativa. Bogotá: ARFO.

Skaalvik, S. y Skaalvik, E. (2004). Gender Differences in Math and Verbal Self-Concept, Performance Expectations, and Motivation. Sex Roles, 50(3), 241-252.

Slootmaeckers, K. (2012). Too afraid to learn?! Attitudes towards statistics as a barrier to learning statistics and acquiring quantitative skills. 4th International Conference on Education and New Learning Technologies. Barcelona: Edulearn12.

Sullivan, G. y Artino, A. (2013). Analyzing and Interpreting Data From Likert-Type Scales. Journal of Graduate Medical Education, 5(4), 541-542.

Tejero, C. y Castro, M. (2011). Validación de la escala de actitudes hacia la estadística en estudiantes españoles de ciencias de la actividad física y del deporte. Revista Colombiana de Estadística, 34(1), 1-14.

Universidad Nacional Autónoma de Honduras. (2014). Informe estadístico: resultados de los procesos de admisión del año 2014. Tegucigalpa: Dirección del Sistema de Admisión de la UNAH. 